\title{
A Primer in Resilience Status for German Medical Graduates - A Necessary Step in Building an Emotionally Equipped Healthcare Workforce
}

Jan Kiesewetter ( $\sim$ jan.kiesewetter@med.Imu.de )

Klinikum der LMU München https://orcid.org/0000-0001-8165-402X

Johanna HUber

Klinikum der LMU München, Institut für Didaktik und Ausbildungsforschung in der Medizin

Research article

Keywords: resilience, stress, burnout, graduates of medical studies

Posted Date: August 31st, 2020

DOI: https://doi.org/10.21203/rs.3.rs-59603/v1

License: (c) (1) This work is licensed under a Creative Commons Attribution 4.0 International License.

Read Full License 


\section{Abstract}

\section{Background}

Resilience is a widely-used catchword in the last couple of years to describe the resistance to psychological strains of life, especially for the healthcare work force. The promises of resilience to burnout sound great and what we all would want: less health impairment despite stress, higher work satisfaction and last but not least higher work performance. However, little is known scientifically regarding the resilience status of the upcoming work-force. With our study we would like to investigate the resilience status of medical graduates from five medical schools within their first year after graduation.

Methods

For the identification of the resilience status we included the 5-point Likert 10-Item Connor-Davidson Resilience Scale, German Version in a graduate survey posted to 5 medical schools and over 1610 eligible participants of whom 610 (60\% female) filled out at least parts of the survey.

Results

The resilience status showed a mean resilience score of $M=37.1(S D=6.30)$. The score ranges from 3.22 ( I am not easily discouraged by failure) to 4.26 ( $\mathrm{am}$ able to adapt to change). The item "I am able to handle unpleasant feeling" is interesting as one third of the participants did choose not to answer. Relationships to other constructs are presented in the article.

\section{Conclusions}

The study shows that the overall resilience status of medical graduates one year after their graduation is rather high, but subjectively they do not feel equivalently resilient for the different aspects they face in their job. Further research needs to see how trainings can provide medical students and professionals with the emotional coping skills that they lack in the moment.

\section{Background}

Resilience is a widely-used catchword in the last couple of years to describe the resistance to psychological strains of life. The promises of resilience to burnout sound great and what we all would want: less health impairment despite stress (Grossman, Niemann, Schmidt, \& Walach, 2004), higher work satisfaction (Shanafelt et al., 2015) and last but not least higher work performance (Loehr, Loehr, \& Schwartz, 2005). However, over many professions little is known scientifically regarding the resilience status of the work-force and if and how resilience can be improved. With our article we would like to investigate the resilience status of medical graduates from five medical schools in Bavaria in their first year after graduation. Before we do so, we will take a look at the scientific constructs related to resilience 
that have been under investigation a lot longer: burnout and stress among medical students, physicians and healthcare staff.

Physician and nursing staff have been found to be prone to burnout (Buser, Schneller, \& Wildgrube, 2007; Schüler \& Dietz, 2004). By employing non-functioning coping mechanisms, physicians have been shown to change their social behavior and their attitudes towards their job and patients (Möller, Laux, Deister, \& Schulte-Körne, 2013; Schüler \& Dietz, 2004). In studies with nurses, a general quality of care impairment could be observed as well as medication errors (Maiden, Georges, \& Connelly, 2011). The reason, again, for this can also be attributed to non-functioning coping mechanisms for difficult situations and emotional blunting. The consequence for burned-out staff and employers are grave and include decreased job performance (Parker \& Kulik, 1995), reduced job commitment (Cherniss, 2016) and stressrelated health problems (Maslach, Schaufeli, \& Leiter, 2001). For medical students a recent review reports burnout rates between $27 \%$ and $75 \%$, a wide range with a lot of regional variance. For Germany no single study was included. A German study with data from 2008 medical students in their final year reported a clinically relevant burnout rate of $20 \%$, a medium burnout score over the whole sample (Koehl-Hackert et al., 2012). The sample has been drawn from one city and since the samples' final year the medical education system in Germany has undergone some changes, so it is hard to say how representative the data is.

In the field of medical education, little is known regarding the coping mechanisms for stress of students. It is known that stress among medical students is rather high but dangerously high stress levels vary between 21\% and 90\% (Fares, Al Tabosh, Saadeddin, El Mouhayyar, \& Aridi, 2016). However, when diagnosing difficult patients showing disruptive behavior, students' diagnostic accuracy was lower than when difficult patients showed friendly behavior (Hege et al., 2018). The reason, again, might lie in nonfunctioning coping mechanisms through which their working memory capacity is impaired; this is an effect that has been long known in cognitive psychological experiments (Eysenck, 1985)

To summarize the results for medical students, physicians and healthcare staff for burnout and stress we have found evidence for what we call the burnout-workload circle. If physicians, medical students and healthcare staff suffer from dangerously high rates of burnout and stress the personnel suffers from higher leave of absence and health related problems. This in itself might reflect back and increases overall workload of the remaining staff and the negative effect continues. Due to high workload, the health care (diagnostic accuracy, time for organizational tasks, time for patients and patient satisfaction) is decreased.

Therefore scientific and public attention has been shifting towards resilience to find ways to avoid burnout and stress before the burnout-workload circle.

Resilience is defined in a multitude of ways; some researchers think of resilience as a sense of flexibility or adaptability (Jackson, Firtko, \& Edenborough, 2007). Others define resilience as an individual's set of skills and attributes that grant them 'the ability to succeed, live, and develop in a positive way despite 
stress or adversity' (Cyrulnik, 2009). Because of resilience's dynamic nature, it differs from related traits such as 'mental toughness' or 'hardiness' (Coulter, Mallett, \& Gucciardi, 2010).

As research shows, resilience can be learned and nurtured through training and practice (Zautra, Hall, \& Murray, 2010). In the realm of healthcare and healthcare providers education, it is especially necessary to obtain and maintain a level of resilience. How can one offer their best services if they are not operating as their best selves? Building a sense of resilience is clearly appealing; higher resilience can lead to a decreased occurrence of burnout (Howe, Smajdor, \& Stöckl, 2012). Resilience also helps to circumvent issues such as impaired concentration, increased cynicism, undermined professional development, and jeopardized care for patients (Dunn, Iglewicz, \& Moutier, 2008).

In German and international medical education little research on resilience in medical students has been done. We have limited knowledge regarding how students' themselves rate their personal resilience in the end of medical school and what factors relate to resilience in medical school. Further, in order to provide trainings on a broader range we need to identify whether students' resilience can indeed be positively influenced.

Therefore, in our study, we aimed to identify medical school graduates' resilience status at the end of their medical education and its relationship to other constructs.

\section{Methods}

\section{Assessment of the resilience status of medical students}

A survey in the course of the so called "The survey of Bavarian medical graduates (Bayerische Absolventenstudie Medizin, MediBAS)" is a multi-centric survey comprised of several validated questionnaires (listed below) conducted in five Bavarian medical schools (Friedrich-Alexander-Universität Erlangen-Nürnberg, Julius-Maximilians-Universität Würzburg, Ludwig-Maximilians-Universität München, Technische Universität München, Universität Regensburg) and one Bavarian veterinary medical school (Ludwig-Maximilians-Universität München). The study was done from October 2018 to January 2019 in collaboration of the Kompetenznetz Medizinlehre Bayern (KMB) and the Bavarian institute for higher education research and higher education planning (Bayerisches Staatsinstitut für Hochschulforschung und Hochschulplanung, IHF). Target persons were those who had finished their final state license examination between 01 . October 2016 and 30. September 2017 at one of the participating faculties. The questionnaire was sent out in an online version (via Questbeck, Globalpark inc.) and a paper-based version. We will present data of the human medical graduates only. 1610 persons were asked to participate, 613 did participate, $60 \%$ were female.

For the identification of the resilience status we included the 10-Item Connor-Davidson Resilience Scale, German Version (Sarubin et al., 2015) in the survey. The 10 items measure resilience with a 5-point Likert type response scale form 0 (never) to 4 (almost always). The questionnaire represents the following aspects of resilience (Connor \& Davidson, 2003): 
- Adapt to change

- Deal with whatever comes my way

- See humorous side of things

- Stress makes me stronger

- Bounce back after illness or injury

- Believe I can achieve goals despite obstacles

- Under pressure I stay focused

- Not easily discouraged by failure

- Think of myself as a strong person when facing challenges

- Able to handle unpleasant feelings

Participants' answers should be given based on reflection from the previous four weeks of their life. The level of agreement within each item allows for the calculation of a score for each participant. Possible overall score ranges vary from a minimum of 0 (lowest level of resilience) to a maximum of 40 (highest level of resilience). We used the German version validated by Sarubin et al. (2015).

To get a more holistic view of what participants' resilience level entails we also included other measures. To self assess professional competence we included the professional competence scale (13 items) of the Freiburger competence questionnaire with overall 45 items (Giesler, Forster, Biller, \& Fabry, 2011). To measure the scientific and communicative competence we included two self-developed scales, whereof scientific competence is based on the learning goals of the national competency based learning objective catalogue medicine (Nationaler Kompetenzbasierter Lernzielkatalog Medizin; wwww.nklm.de). We further asked questions regarding their demography, income and working time, discrimination and mobbing experiences as well as their perception of how they deal with excessive demands, how they handle feedback and workplace learning. The latter two measures are adapted from a questionnaire for social workers (Kyndt, Govaerts, Verbeek, \& Dochy, 2013).

\section{Results}

\section{Resilience of medical students and its relationship to other constructs}

The Connor-Davidson resilience scale resulted in a mean resilience score of $M=37 \cdot 1(S D=0,63)$. Resilience was measured highly reliable (Cronbachs $a=.88$ ). We have depicted the individual scores in Table 1. 
Table 1

Resilience status scores of medical students

\begin{tabular}{|llll|}
\hline I am able to... & N & M & SD \\
\hline Adapt to change & 572 & 4.26 & 0.77 \\
\hline Deal with whatever comes my way & 571 & 3.43 & 0.91 \\
\hline See humorous side of things & 569 & 3.41 & 1.03 \\
\hline Stress makes me stronger & 570 & 3.46 & 0.94 \\
\hline Bounce back after illness or injury & 571 & 4.06 & 0.96 \\
\hline Believe I can achieve goals despite obstacles & 568 & 4.14 & 0.85 \\
\hline Under pressure I stay focused & 569 & 3.60 & 0.90 \\
\hline Not easily discouraged by failure & 567 & 3.22 & 0.99 \\
\hline Think of myself as a strong person when facing challenges & 567 & 3.78 & 0.91 \\
\hline Able to handle unpleasant feelings & 418 & 3.78 & 0.89 \\
\hline Overall score sum & & 37.10 & 6.3 \\
\hline
\end{tabular}

The score of the last item is interesting as many of the participants did choose not to answer. In general the scores seem relatively high compared to other nonclinical samples (Sarubin et al., 2015).

To further advance the knowledge on the resilience status we calculated the relationship of the scale to other measurements of the survey with Pearson correlations. Resilience status did positively relate to estimated self-assessment of communication competence (12 items, correlation of $r=.18 ; p<.05)$ and professional competence (13 items, correlation of $r=.19 ; p<.05)$ and scientific competence (10 items, correlation $r=.19 ; p<.05)$. Interestingly, a significant negative relation between resiliency and the question how satisfied participants were with their first job $(r=-.18 ; p<.01)$ was found. Conversely, participants' satisfaction with their medical education did relate to their resiliency scores positively $(r=.12, p<.01)$. There was a negative significant correlation regarding participants' subjective excessive demands (How does the following attribute apply to you: professionally overwhelmed; $r=-.20 ; p<.01$; workload $r=-.10$; $p<.05$; too many stand-by duties $r=-.09 ; p<.05$ ). Further, we found that participants scores' regarding discrimination and mobbing experiences regarding their ethnicity was significantly related to resilience scores $(r=.08, p<.05)$

Interestingly, the resiliency score did not relate significantly to scores of professional workplace learning $(r=.08, n . s$.$) and feedback (r=.01$, n.s.). The resiliency score did not relate significantly to participants' income $(r=.04, n . s$.$) , nor their contractual (r=.04$, n.s.) or real working time $(r=.05$, n.s.). The results are summarized in Table 2. 
Table 2

The results of the relationship of medical students' resilience status.

Presented are the Pearson correlations to the overall resilience scores.

\begin{tabular}{|lcc|}
\hline Scales & $\mathbf{r}$ & $\mathbf{p}$ \\
\hline Communication competence & .18 & $<.05$ \\
\hline Professional competence & .19 & $<.05$ \\
\hline Scientific competence & .19 & $<.05$ \\
\hline Feedback & .01 & n.s. \\
\hline Professional workplace learning & .08 & n.s. \\
\hline Singular items & $r$ & p \\
\hline How satisfied were you with your first job? & -.18 & $<.01$ \\
\hline How satisfied were you with your medical education? & .12 & $<.01$ \\
\hline Excessive demand: professionally overwhelmed & -.20 & $<.01$ \\
\hline Excessive demand: workload & -.10 & $<.05$ \\
\hline Excessive demand: too many stand-by duties & -.09 & $<.05$ \\
\hline Discrimination experiences regarding ethnicity? & .08 & $<.05$ \\
\hline Participants' income & .04 & n.s. \\
\hline Contractual working hours & .04 & n.s. \\
\hline Actual working hours & .05 & n.s. \\
\hline
\end{tabular}

\section{Discussion}

The study is an important primer on resilience in medical school, but many questions remain unanswered. The resilience status of medical students after their graduation has been investigated in our study. Our results indicate that medical graduates in their first year after graduation do estimate their resilience to be rather high. Further the scores indicate that participants' resilience scores vary. Especially, their estimate regarding discouragement by failure is rather low and over 150 of the participants who answered all other questions chose not to answer whether they are able to handle unpleasant feelings. Medicine does entail negative feelings for physicians and patients (Ofri, 2013) and it is one of the core components of a resilient healthcare workforce to deal with failure (Wears \& Wu, 2002). More research is needed in order to investigate which coping mechanisms graduates and medical students have in order to create evidence regarding the external validity of their estimate. The resilience status we assessed did provide some insight regarding the relationship of resilience in a broader context. As could be expected a higher level of professional, communicative and scientific competences relates to higher levels of resilience and vice versa. Further participants who felt their job demand to be more excessive did have 
lower resiliency scores or vice versa less resilient participants felt the job demand to be higher. Interestingly, no relation to their actual and contractual weekly working hours was found. This provides evidence that indeed it is not the actual workload that is important, but the way how physicians cope with the workload.

The negative relationship of job satisfaction and resilience can point towards the idea that resilience is built from necessity to develop it, same goes for the negative relationship to discrimination against ethnicity. Of course this is cause for concern for medical schools and medical educators should strive towards a more common and standardized implementation of resiliency in their medical school and not wait for students or physicians to have negative experiences in order to develop resiliency. As there was no relationship to feedback at the workplace, workplace-based learning and the resilience score the continuing medical education put in place does not seem to suffice to advance physicians' resilience. Specialized resiliency education might help to build resiliency in undergraduate and continuing medical education (Tempski, Martins, \& Paro, 2012). In our manualized training program we focus on the basics of energy management, but go beyond these to provide students with a sense of how to deal with emotions and reflect upon their achievement emotions. More research is needed to assess the coping mechanisms we did train and short- and long-term effects of resilience training.

With a growing need and possibility to train medical students there is also a growing need for providing educators with the tools how to facilitate resiliency trainings. We know little of resilience of staff in higher education (Gold, 1984) and even less regarding their ability to teach courses with content derived from cognitive behavioral group therapy. There is a need to provide faculty with teaching skills to manage and deal with emotionally activated groups, despite being non-clinicians. Emotionally activated groups means that some of the course contents is explicitly designed trigger emotional responses necessary to reflect and change ones behavior. Yet, it should be made certain that teachers can deal with these responses and know if and when to offer further help or guidance where to seek help. Resilience trainings that we offer are facilitated by experienced trainers with a psychotherapy and group psychotherapy background. Knowledge to other (medical) specialist educators needed could be provided in train the trainer workshops.

Not only in medical education, but also in other fields of higher education there is a growing need to embed resilience trainings. Many of the parts of the trainings can easily be taught or could be taught if adapted to other specializations. Especially teacher education, social workers, kindergarten teachers or other specializations in the human services industry known to be prone to burnout (Burke \& Greenglass, 1988; Chang, 2009) could benefit from the training.

\section{Limitations}

Regarding the resilience status of medical graduates we utilized a sample from graduates from Bavarian medical schools and we can't guarantee for their representativeness, neither for medical students nor for medical graduates worldwide. However, with a large response rate of over 600 participants it is the best 
sample for Germany and the largest sample for medical schools worldwide that assess resilience that we know of. We have provided correlations for resilience with various other measurements from the survey and of course correlation does not answer questions regarding causation. Nonetheless, we are only beginning to understand resilience in medical school and the resilience status presented here has provided us with an idea where to look at in further research.

\section{Conclusions}

For medicine, by gaining increased insight into resilience status. We need to take the advancement of individualized emotional coping mechanisms of medical students and healthcare professionals seriously (Howe Smajdor \& Stöckl, 2012). In turn, greater resilience and functioning coping strategies could lead to better diagnostic accuracy, decreased medical assessments and laboratory procedures, and fewer cases of physician burnout. In short, building this practice in more medical schools is beneficial not only for practitioners, but also for patients.

\section{Abbreviations}

MediBAS - Bayerische Absolventenstudie Medizin

KMB - Kompetenznetz Medizinlehre Bayern

IHF - Bavarian institute for higher education research and higher education planning

\section{Declarations}

Ethics approval and consent to participate

All participants gave consent to participate.

The ethics committee of the LMU Hospital Munich (Proposal 18-535) approved the study of the assessment of the resilience status. All study participants signed an informed consent. The informed consent signed by all study participants included a consent of publication.

Consent for publication

All authors gave consent for publication.

Availability of data and materials

The MediBAS data can be requested from the Bavarian Institute of Higher Education Research and Planning (www.ihf.bayern.de)

Competing interests 
None of the authors have any competing interests to declare

Funding

The study was not funded

Authors' contributions

$\mathrm{JH}$ was involved in the design of the study, the acquisition of the data and the drafting of this article JK was involved in the analysis of the data and the drafting of this article

All authors have read and approved the manuscript.

Authors' information

$\mathrm{JH}$ is a post-Doctoral researcher and responsible for evaluation and faculty development

JK is an associate professor and psychotherapist and involved in resilience training and research

Acknowledgements

We thank all responsible people and partners of the MediBAS project for their friendly cooperation and the transfer of the research data. In this context we would like also to thank the participants of the MediBAS.

\section{References}

Burke RJ, Greenglass ER. Career orientations and psychological burnout in teachers. Psychological reports. 1988;63(1):107-16.

Buser K, Schneller T, Wildgrube K. (2007). Medizinische Psychologie, medizinische Soziologie: Kurzlehrbuch zum Gegenstandskatalog: Elsevier, Urban \& FischerVerlag.

Chang M-L. An appraisal perspective of teacher burnout: Examining the emotional work of teachers. Educational psychology review. 2009;21(3):193-218.

Cherniss C. (2016). Beyond burnout: Helping teachers, nurses, therapists and lawyers recover from stress and disillusionment: Routledge.

Connor KM, Davidson JR. Development of a new resilience scale: The Connor-Davidson resilience scale (CD-RISC). Depress Anxiety. 2003;18(2):76-82.

Coulter TJ, Mallett CJ, Gucciardi DF. Understanding mental toughness in Australian soccer: Perceptions of players, parents, and coaches. J Sports Sci. 2010;28(7):699-716. 
Cyrulnik B. Resilience: How to Gain Strength from Childhood Adversity. London: Penguin; 2009.

Dunn LB, Iglewicz A, Moutier C. A conceptual model of medical student well-being: promoting resilience and preventing burnout. Acad Psychiatry. 2008;32(1):44-53.

Eysenck MW. Anxiety and cognitive-task performance. Personality Individ Differ. 1985;6(5):579-86.

Fares J, Al Tabosh H, Saadeddin Z, El Mouhayyar C, Aridi H. Stress, burnout and coping strategies in preclinical medical students. North American journal of medical sciences. 2016;8(2):75.

Giesler M, Forster J, Biller S, Fabry G. (2011). Entwicklung eines Fragebogens zur Erfassung von Kompetenzen in der Medizin: Ergebnisse zur Reliabilität und Validität. GMS Z Med Ausbild, 28(2), Doc31.

Gold Y. (1984). Burnout: A major problem for the teaching profession. Education, 104(3).

Grossman P, Niemann L, Schmidt S, Walach H. Mindfulness-based stress reduction and health benefits: A meta-analysis. J Psychosom Res. 2004;57(1):35-43.

Howe A, Smajdor A, Stöckl A. Towards an understanding of resilience and its relevance to medical training. Medical education. 2012;46(4):349-56.

Jackson D, Firtko A, Edenborough M. Personal resilience as a strategy for surviving and thriving in the face of workplace adversity: a literature review. J Adv Nurs. 2007;60(1):1-9.

Koehl-Hackert N, Schultz J-H, Nikendei C, Möltner A, Gedrose B, van den Bussche H, Jünger J. (2012). Belastet in den Beruf-Empathie und Burnout bei Medizinstudierenden am Ende des Praktischen Jahres. Zeitschrift für Evidenz, Fortbildung und Qualität im Gesundheitswesen, 106(2), 116-124.

Kyndt E, Govaerts N, Verbeek E, Dochy F. Development and validation of a questionnaire on informal workplace learning outcomes: A study among socio-educational care workers. The British Journal of Social Work. 2013;44(8):2391-410.

Loehr J, Loehr JE, Schwartz T. (2005). The power of full engagement: Managing energy, not time, is the key to high performance and personal renewal: Simon and Schuster.

Maiden J, Georges JM, Connelly CD. Moral distress, compassion fatigue, and perceptions about medication errors in certified critical care nurses. Dimens Crit Care Nurs. 2011;30(6):339-45.

Maslach C, Schaufeli WB, Leiter MP. Job burnout. Ann Rev Psychol. 2001;52(1):397-422.

Thieme Stuttgart

Psychiatrie, Psychosomatik und Psychotherapie 
Möller H-J, Laux G, Deister A, Schulte-Körne G. (2013). Psychiatrie, Psychosomatik und Psychotherapie: Thieme Stuttgart.

Ofri D. (2013). What doctors feel: how emotions affect the practice of medicine: Beacon Press.

Parker PA, Kulik JA. Burnout, self-and supervisor-rated job performance, and absenteeism among nurses. J Behav Med. 1995;18(6):581-99.

Sarubin N, Gutt D, Giegling I, Bühner M, Hilbert S, Krähenmann O,.. . Rujescu D. (2015). Erste Analyse der psychometrischen Eigenschaften und Struktur der deutschsprachigen 10-und 25-Item Version der ConnorDavidson Resilience Scale (CD-RISC). Zeitschrift für Gesundheitspsychologie.

Schüler J, Dietz F. (2004). Kurzlehrbuch Medizinische Psychologie und Soziologie: Georg Thieme Verlag.

Shanafelt TD, Hasan O, Dyrbye LN, Sinsky C, Satele D, Sloan J, West CP. (2015). Changes in burnout and satisfaction with work-life balance in physicians and the general US working population between 2011 and 2014. Paper presented at the Mayo Clinic Proceedings.

Tempski P, Martins MA, Paro HB. Teaching and learning resilience: a new agenda in medical education. Medical Education-Oxford. 2012;46(4):345.

Wears RL, Wu AW. Dealing with failure: the aftermath of errors and adverse events. Ann Emerg Med. 2002;39(3):344-6.

Zautra AJ, Hall JS, Murray KE. (2010). A new definition of health for people and communities. Handbook of adult resilience, 1 .

Notes.

The survey of Bavarian medical. graduates, the Bayerische Absolventenstudie Medizin (MediBAS) was carried out in the context of the working group Qualitätsmanagement und Absolventenbefragungen of the Kompetenznetz Medizinlehre Bayern, in cooperation with the Bayerisches Staatsinstitut für Hochschulforschung und Hochschulplanung (IHF). 